\title{
Lehrbuch
}

\section{der Palliativmedizin}

Aulert E, Nauck F Radbruch L (Hrsg.). Lehrbuch der Palliativmedizin. 2. überarb. u. erw. Auflage 2007. XXVII, 1423 Seiten mit 225 z. T. farb. Abb.; EUR 129,00; ISBN 978-3-7945-2361-0

Inhalt:

- Grundlage (Definition, Ethik, Organisationsformen)

- Symptombehandlung inklusive Schmerztherapie

- Spezielle Palliativmedizin

- Onkologie

- Aids

- Spezielle internistische Erkrankungen

- Geriatrie

- Pädiatrie

Palliativpflege:

- Sterbephase

- Psychosoziale Aspekte

- Spirituelle Begleitung

- Trauer und Trauerbewältigung

- Rehabilitation

- Dokumentation und Qualifikation

- Aus- und Weiterbildung und Forschung

- Besondere Hinweise

Berücksichtigt man die umfassende Definition von Palliativmedizin, die im vergangenen Jahr in Wiesbaden auf dem Kongress der Deutschen Gesellschaft für Palliativmedizin überarbeitet und beschlossen wurde, kann ein Lehrbuch der Palliativmedizin nur ein ganzheitliches Konzept haben. So ist dieses bereits zum Standardwerk avancierte Lehrbuch zwangsläufig umfassend und umfangreich. Hierzu hat sich der bisherige Herausgeber und erfahrene Berliner Palliativmediziner Aulbert die nicht minder kompetenten Lehrstuhlinhaber Friedemann Nauck, Göttingen, und Lucas Radbruch, Aachen, zur Seite genommen und Ihnen für das Buch ihre eigene hochkompetente Handschrift überlassen.

Das Buch enthält - wie auch andere bereits auf dem Markt befindliche einschlägige Kurzlehrbücher - praktische Handlungsanweisungen, insbesondere aber auch ausführliche Grundlageninformationen. Diese sind aktualisiert und theoretisch fundiert, sprechen aber aus der und an die Seele der Palliativmedizin. Der schöne Beitrag des Theologen Weiher zum Thema „Subproblematik der Spiritualität in der Palliativmedizin“ sei hierfür einmal als Beispiel besonders hervorgehoben. Ein in jeder Hinsicht empfehlenswertes Buch für alle an der Palliativmedizin interessierten Berufsgruppen. Seinen Preis ist es allemal wert.

Prof. Dr. med. Winfried Hardinghaus, Osnabrück 